\title{
A New Modification of the Differential Transform Method for a SIRC Influenza Model
}

\author{
S.F.M.IBRAHIM \\ 1-King Abdulaziz University, \\ Faculty of science For North Jeddah, \\ Dept. of Mathematics, Jeddah, Saudi Arabia., \\ 2-Ain Shams University, Faculty of Education, \\ Department of Mathematics, Roxy, Cairo, Egypt.
}

\author{
S. M. ISMAIL \\ Misr University For Science \&Technology \\ Faculty of Engineering, \\ Dept. of Basic science, \\ 6Th of October City, Egypt.
}

\begin{abstract}
In this paper, approximate analytical solution of SIRC model associated with the evolution of influenza A disease in human population is acquired by the modified differential transform method (MDTM). The differential transform method (DTM) is mentioned in summary. MDTM can be obtained from DTM applied to Laplace, inverse Laplace transform and padé approximant. The MDTM is used to increase the accuracy and accelerate the convergence rate of truncated series solution getting by the DTM. The analytical-numerical technique can be used in order to produce simulations with different initial conditions, parameter values for different values of the basic reproduction number.
\end{abstract}

\section{General Terms}

Cross-immunity, SIR model, Laplace transform, Influenza, Differential transformation method

\section{Keywords}

SIRC model, Epidemic models, Modified Differential transformation method, padé approximants.

\section{INTRODUCTION}

Influenza is caused by a virus that attacks mainly the upper respiratory tract, nose, throat and bronchi and rarely also the lungs. Many people recover within 1-2 weeks without requiring any medical treatment. In the very young, the elderly and people suffering from medical conditions such as lung diseases, diabetes, cancer, kidney or heart problems, influenza poses a serious risk. In these people, the infection may lead to severe complications of underlying diseases, pneumonia and death. In annual influenza epidemics 5-15\% of the populations are affected with upper respiratory tract infections. Hospitalization and deaths mainly occur in high risk groups (elderly, chronically ill). Annual epidemics are possibly between three and five million cases of severe illness and between 250,000 and 500,000 deaths every year around the world. Influenza is transmitted by a virus that can be of three different types, namely A, B and C [1]. Among these taypes, the virus $\mathrm{A}$ is epidemiologically the most important one for human beings, because it can recombine its genes with those of strains circulating in animal populations such as birds, swine, horses etc. Unfortunately, within type a virus, there are several subtypes, $\mathrm{H} 1 \mathrm{~N} 1, \mathrm{H} 3 \mathrm{~N} 2, \mathrm{H} 5 \mathrm{~N} 1$, etc., each one of these has been pointed as the causal of recent pandemics. Much evidence shows that the antigenic distance between two different strains influences the degree of partial immunity, often called cross-immunity, conferred to a host already infected by one of the strains with respect to the other[2]. Mathematical models have proven to be useful tools to study the dynamics of viral infections, within these models, compartmental models have been transformed of ordinary or partial differential equations. Over the last two decades, a number of epidemic models for predicting the spread of influenza through human population have been proposed based on either the classical susceptible-infected-removed (SIR) model developed by Kermack and McKendrick[3]. Casagrandi et al.[2] have introduced SIRC model by adding a new compartment $\mathrm{C}$, which can be called cross-immune compartment, to the SIR model. This cross-immune compartment (C) describes an intermediate state between the fully susceptible (S) and the fully protected (R)one. They have studied the dynamical behaviors of this model numerically [4]. Jodar et al. [5] developed two nonstandard finite difference schemes to obtain numerical solutions of a influenza A disease model presented by Casagrandi et al.[2] Very recently Samanta[4] considered a nonautonomous SIRC epidemic model for Influenza A with varying total population size and distributed time delay. This model assumes no immune interference between the different A virus subtypes, that is why they only considered one virus subtype. In this paper, the modified differential transform method (MDTM) has been applyed will be employed in a straightforward manner without any need of linearization or smallness assumptions. DTM was first applied in the engineering domain by [6]. DTM provides an efficient explicit and numerical solution with high accuracy, minimal calculations, sparing of physically unrealistic assumptions. However, DTM has some drawbacks. By using DTM, a series solution which is obtained, in practice a truncated series solution. This series solution does not exhibit the periodic behavior which is characteristic of oscillator equations and gives a good approximation to the true solution in a very small region. In order to develop the accuracy of DTM, an alternative technique is used which modifies the series solution for nonlinear oscillatory systems as follows: first apply the Laplace transformation to the truncated series obtained by DTM, then convert the transformed series into a meromorphic function by forming its Padé approximants([7],[8],[9],[10],[11]), and finally accept an inverse Laplace transform to obtain an analytic solution, which may be periodic or a better approximation solution than the DTM truncated series solution.

\section{The SIRC MODEL}

Casagrandi et al. [2] considered the model

$\frac{d S}{d t}=\mu(1-S)+\gamma C-\beta S I$, 


$$
\begin{aligned}
& \frac{d I}{d t}=\beta S I+\sigma \beta S I-(\mu+\theta) I, \\
& \frac{d R}{d t}=(1-\sigma) \beta C I+\theta I-(\mu+\delta) R, \\
& \frac{d C}{d t}=\delta R-\beta C I-(\mu+\gamma) C,
\end{aligned}
$$

With initial conditions

$S(0)=M_{1}, I(0)=M_{2}, R(0)=M_{3}, C(0)=M_{4}$. Where

$\mu$ is the mortality rate,

$\theta$ is the rate of progression from infective to recovered per year,

$\delta$ is the rate of progression from recovered to cross-immune per year,

$\gamma$ is the rate of progression from recovered to susceptible per year,

$\sigma$ is the recruitment rate of cross-immune into the infective,

$\beta$ is the contact rate per year.

The disease free equilibrium is locally asymptotically stable if and only if

$\frac{\beta}{\mu+\theta}<1$

and unstable if

$\frac{\beta}{\mu+\theta}>1$

There exists a unique and positive endemic equilibrium point if and only if $(\beta /(\mu+\theta))>1$ which is locally asymptotically stable under some conditions on the coefficients[12]

\section{PADÉ APPROXIMATIONS}

Some techniques exist to increase the convergence of a given series. Among them, the so- called padé technique is widely applied in this section the notion of rational approximations is introduced for functions. The function $\mathrm{f}(\mathrm{x})$ will be approximated over a small portion of its domain. For example, if $f(x)=\cos (x)$, it is sufficient to have a formula to generate approximations on the interval $[0, \pi / 2]$. Then trigonometric identities can be used to compute $\cos (\mathrm{x})$ for any value $\mathrm{x}$ that lies outside $[0, \pi / 2]$. A rational approximation to

$\mathrm{f}(\mathrm{x})$ on $[\mathrm{a}, \mathrm{b}]$ is the quotient of two polynomials $P_{N}(x)$ and $Q_{M}(x)$ $[\mathrm{N} / \mathrm{M}](\mathrm{x})$ can be used to denote this quotient:

$$
[N / M](x)=\frac{P_{N}(x)}{Q_{M}(x)} \text { for } a \leq x \leq b .
$$

Our goal is to make the maximum error as small as possible. For a given amount of computational effort, one can usually construct a rational approximation that has a smaller overall error on $[a, b]$ than a polynomial approximation. The development is an introduction and will be limited to Padé approximations. The method of Padé requires that $\mathrm{f}(\mathrm{x})$ and its derivative be continuous at $\mathrm{x}=0$. There are two reasons for the arbitrary choice of $x=0$. First, it makes the manipulations simpler. Second, a change of variable can be used to shift the calculations over to an interval that contains zero. The polynomials used in Eq. (5) are

$P_{N}(x)=p_{0}+p_{1} x+p_{2} x^{2}+\ldots+p_{N} x^{N}$

And

$Q_{M}(x)=1+q_{1} x+q_{2} x^{2}+\ldots+q_{M} x^{M}$

The polynomials in (6) and (7) are constructed so that $f(x)$ and $[N / M](x)$ agree at $x=0$ and their derivatives up to $N+M$ agree at $x=0$. In the case $Q_{0}(x)=1$, the approximation is just the Maclaurin expansion for $\mathrm{f}(\mathrm{x})$. For a fixed value of $\mathrm{N}+\mathrm{M}$ the error is smallest when $P_{N}(x)$ and $Q_{M}(x)_{\text {have the }}$ same degree or when $P_{N}(x)_{\text {has degree one higher than }}$ $Q_{M}(x)$. Notice that the constant coefficient of $Q_{M}$ is $\mathrm{q}_{0}=1$. This is permissible, because it cannot be 0 and $[\mathrm{N} / \mathrm{M}]$ (x) is not changed when both $P_{N}(x)$ and $Q_{M}(x)$ are divided by the same constant. Hence the rational function $[\mathrm{N} / \mathrm{M}](\mathrm{x})$ has $\mathrm{N}+\mathrm{M}+1$ unknown coefficients. Assume that $\mathrm{f}(\mathrm{x})$ is analytic and has the Maclaurin expansion

$f(x)=a_{0}+a_{1} x+a_{2} x^{2}+\ldots+a_{k} x^{k}+\ldots$,

And form the difference

$$
\begin{aligned}
& f(x) Q_{M}(x)-P_{N}(x)=z(x): \\
& \left(\sum_{j=0}^{\infty} a_{j} x^{j}\right)\left(\sum_{j=0}^{M} q_{j} x^{j}\right)-\sum_{j=0}^{N} p_{j} x^{j}=\left(\sum_{j=N+M+1}^{\infty} c_{j} x^{j}\right)
\end{aligned}
$$

The lower index $\mathrm{j}=\mathrm{M}+\mathrm{N}+1$ in the summation on the right side of (9) is chosen because the first $N+M$ derivatives of $f(x)$ and $[N / M](x)$ are to agree at $x=0$. When the left side of (9) is multiplied out and the coefficients of the powers of $x^{j}$ are set equal to zero for $\mathrm{k}=0,1, \ldots, \mathrm{N}+\mathrm{M}$, the result is a system of $\mathrm{N}+\mathrm{M}+1$ linear equations:

$a_{0}-p_{0}=0$

$q_{1} a_{0}+a_{1}-p_{1}=0$

$q_{2} a_{0}+q_{1} a_{1}+a_{2}-p_{2}=0$

$q_{3} a_{0}+q_{2} a_{1}+q_{1} a_{2}+a_{3}-p_{3}=0$

$q_{M} a_{N-M}+q_{M-1} a_{N-M+1}+\ldots+a_{N}-p_{N}=0$ 
$q_{M} a_{N-M+1}+q_{M-1} a_{N-M+2}+\ldots+q_{1} a_{N}+a_{N+1}=0$

$q_{M} a_{N-M+2}+q_{M-1} a_{N-M+3}+\ldots+q_{1} a_{N+1}+a_{N+2}=0$

$q_{M} a_{N}+q_{M-1} a_{N+1}+\ldots+q_{1} a_{N+M-1}+a_{N+M}=0$

Notice that in each equation the sum of the subscripts on the factors of each product is the same, and this sum increases consecutively from 0 to $\mathrm{N}+\mathrm{M}$. The $\mathrm{M}$ equations in (11)

involve only the unknowns $q_{1}, q_{2}, \ldots q_{M}$ and must be solved first. Then the equations in (10) are used successively to find $P_{0}, p_{1}, \ldots, p_{N}$

\section{BASIC DEFINITIONS OF DIFFERENTIAL TRANSFORMATION METHOD}

Pukhov [13] proposed the concept of differential transformation, where the image of a transformed function is computed by differential operations, which is different from the traditional integral transforms as are Laplace and Fourier. Thus, this method becomes a numerical-analytic technique that formalizes the Taylor series in a totally different manner. Differential transformation is a computational method that can be used to solve linear (or non-linear) ordinary (or partial) differential equations with their corresponding boundary conditions. A pioneer using this method to solve initial value problems is Zhou [6], who introduced it in a study of electrical circuits. Additionally, differential transformation has been applied to solve a variety of problems that are modeled with differential equations ([14],[15],[16],[17])

The method consists of, given system of differential equations and related initial conditions; these are transformed into a system of recurrence equations that finally leads to a system of algebraic equations whose solutions are the coefficients of a power series solution.

For the sake of clarity in the presentation of the DTM and in order to help to the reader we summarize the main issues of the method that may be found in [6].

Definition 4.1 A differential transformation $\mathrm{Y}(\mathrm{k})$ of function $\mathrm{y}(\mathrm{x})$ is defined as follows [18]

$Y(k)=\frac{1}{k !}\left[\frac{d^{k} y(x)}{d x^{k}}\right]_{x=0}$

In (12), $y(x)$ is the Original function and $\mathrm{Y}(\mathrm{k})$ is the transformed function. Differential inverse transform of $\mathrm{Y}(\mathrm{k})$ is defined as follows

$$
y(x)=\sum_{k=0}^{\infty} x^{k} Y(k)
$$

In fact. From (12) and (13), we obtain

$$
y(x)=\sum_{k=0}^{\infty} \frac{x^{k}}{k !}\left[\frac{d^{k} y(x)}{d x^{k}}\right]_{x=0}
$$

Equation (14) implies that the concept of differential transformation is derived from the Taylor series expansion.
From Equation (12) and (13), it is easy to obtain the following mathematical operations:

1. If $y(x)=g(x) \pm h(x)$ then

$Y(k)=G(k) \pm H(k)$.

2. If $y(x)=c g(x)$ then $Y(k)=c G(k)$, c is a constant.

3. If $y(x)=\frac{d^{n} g(x)}{d x^{n}}$, then

$Y(k)=\frac{(k+n)}{k !} G(k+n)$.

4. If $y(x)=g(x) h(x)$ then

$Y(k)=\sum_{l=0}^{k} G(l) H(k-l)$.

5. If $y(x)=x^{n}$ then

$Y(k)=\delta(k-n)=\left\{\begin{array}{l}1, k=n \\ 0, \mathrm{k} \neq \mathrm{n}\end{array}, \delta\right.$ is the

Kronecker delta

6. If $y(x)=u(x) v(x) w(x)$ then

$Y(k)=\sum_{s=0}^{k} \sum_{m=0}^{k-s} U(s) V(m) W(k-s-m)$.

\subsection{THE OPERATION PROPERTIES OF DIFFERENTIAL TRANSFORMATION}

If $\mathrm{x}(\mathrm{t})$ and $\mathrm{y}(\mathrm{t})$ are two uncorrelated functions with time $\mathrm{t}$ where $X(k)$ and $Y(K)$ are the transformed functions corresponding to $\mathrm{x}(\mathrm{t})$ and $\mathrm{y}(\mathrm{t})$ then the fundamental mathematics operations can be proved by differential Transformation and are listed as follows [16]:(1) Linearity. If $X(k)=D[x(t)], \quad Y(k)=D[y(t)]$ and $c_{1}$ and $c_{2}$ are independent of $\mathrm{t}$ and $\mathrm{k}$ then

$$
D\left[c_{1} x(t) \pm c_{2} y(t)\right]=c_{1} X(k) \pm c_{2} Y(k)
$$

Thus, if $\mathrm{c}$ is a constant, Then $D[c]=c \delta(k)$, where $\delta$ is the kronecer delta function.

(2) Convolution. if

$$
\begin{aligned}
& z(t)=x(t) y(t), x(t)=D^{-1}[X(k)], \\
& y(t)=D^{-1}[Y(k)]
\end{aligned}
$$
and $\otimes$

denote the convolution and Symbol D denoting the differential transformation process. Then 
$D[z(t)]=D[x(t) y(t)]$

$=X(k) \otimes Y(k)$

$=\sum_{l=0}^{k} x(l) Y(k-l)$

If $y(x)=y_{1}(x) y_{2}(x) \ldots y_{n-1}(x) y_{n}(x)$ then

$Y(k)=\sum_{k_{n-1}}^{k} \sum_{k_{n-2}}^{k_{n-1}} \ldots \sum_{k_{2}}^{k_{3}} \sum_{k_{1}=0}^{k_{2}} Y_{1}\left(k_{1}\right)$

$\times Y_{2}\left(k_{2}-k_{1}\right) . . Y_{n-1}\left(k_{n-1}-k_{n-2}\right)$

$\times Y_{n}\left(k-k_{n-1}\right)$

The proof of above properties is deduced from the definition of the differential transform

\section{APPLICATION OF THE SIRC INFIUENZA MODEL}

In this section, the differential transformation technique is applied to solve nonlinear differential equations system such as SIRC influenza model. The following recurrence relation to the SIRC influenza model is obtained By using the fundamental operations of differential transformation method. Applying this method, the system in equations (1)-(4) can be written as follows:

$$
\begin{aligned}
& \tilde{S}(k+1)=\frac{1}{k+1}\left[\mu(\delta(k)-\tilde{S}(k))-\beta \sum_{l=0}^{k} \tilde{S}(l) \tilde{I}(k-l)+\gamma \tilde{C}(k)\right]_{(18)} \\
& \tilde{I}(k+1)=\frac{1}{k+1}\left[\beta \sum_{l=0}^{k} \tilde{S}(l) \tilde{I}(k-l)+\sigma \beta \sum_{l=0}^{k} \tilde{C}(l) \tilde{I}(k-l)-(\mu+\theta) \tilde{I}(k)\right]_{(19)} \\
& \tilde{R}(k+1)=\frac{1}{k+1}\left[(1-\sigma) \beta \sum_{l=0}^{k} \tilde{C}(l) \tilde{I}(k-l)+\theta \tilde{I}(k)-(\mu+\delta) \tilde{R}(k)\right]_{(20)} \\
& \tilde{C}(k+1)=\frac{1}{k+1}\left[\delta \tilde{R}(k)-\beta \sum_{l=0}^{k} \tilde{C}(l) \tilde{I}(k-l)-(\mu+\gamma) \tilde{C}(k)\right]_{(21)}
\end{aligned}
$$$$
\text { with } \tilde{S}(0)=M_{1}, \tilde{I}(0)=M_{2}, \tilde{R}(0)=M_{3}, \tilde{C}(0)=M_{4} \text {. }
$$

Are differential transform of respectively.

$$
S(t), I(t), R(t), C(t)
$$

Thus, from a process of inverse differential transformation, it can be obtained the solutions in the power series

$$
\begin{aligned}
& S(t)=\sum_{k=0}^{n} \tilde{S}(k) t^{k}, \\
& I(t)=\sum_{k=0}^{n} \tilde{I}(k) t^{k}, \\
& R(t)=\sum_{k=0}^{n} \tilde{C}(k) t^{k}, \\
& C(t)=\sum_{k=0}^{n} \tilde{C}(k) t^{k},
\end{aligned}
$$

Therefore

$$
\begin{aligned}
& S(t)=M_{1}+\left(-M_{1} M_{2} \beta+M_{4} \gamma+\left(1-M_{1}\right) \mu\right) t+ \\
& \frac{1}{2}\left[\begin{array}{l}
\mu\left(M_{1} M_{2} \beta-M_{4} \gamma-\left(1-M_{1}\right) \mu\right) \\
+\gamma\left(-M_{2} M_{4} \beta+M_{3} \delta-M_{4}(\gamma+\mu)\right) \\
-\beta\left(\begin{array}{l}
M_{2}\left(-M_{1} M_{2} \beta+M_{4} \gamma+\left(1-M_{1}\right) \mu\right) \\
+M_{1}\left(M_{1} M_{2} \beta-M_{2}(\theta+\mu)+M_{2} M_{4} \beta \sigma\right)
\end{array}\right)
\end{array}\right] t^{2}
\end{aligned}
$$$$
I(t)=M_{2}+\left(M_{1} M_{2} \beta-M_{2}(\theta+\mu)+M_{2} M_{4} \beta \sigma\right) t+
$$$$
\frac{1}{2}\left[\begin{array}{c}
-(\theta+\mu)\left(M_{1} M_{2} \beta-M_{2}(\theta+\mu)+M_{2} M_{4} \beta \sigma\right)+ \\
\beta\left(\begin{array}{l}
M_{2}\left(-M_{1} M_{2} \beta+M_{4} \gamma+\left(1-M_{1}\right) \mu\right) \\
+M_{1}\left(M_{1} M_{2} \beta-M_{2}(\theta+\mu)+M_{2} M_{4} \beta \sigma\right)
\end{array}\right) \\
+\beta \sigma\left(\begin{array}{l}
M_{2}\left(-M_{2} M_{4} \beta+M_{3} \delta-M_{4}(\gamma+\mu)\right) \\
+M_{4}\left(M_{1} M_{2} \beta-M_{2}(\theta+\mu)+M_{2} M_{4} \beta \sigma\right)
\end{array}\right)
\end{array}\right] t^{2}
$$

$R(t)=M_{3}+\left(M_{2} \theta-M_{3}(\delta+\mu)+M_{2} M_{4} \beta(1-\sigma)\right) t+$

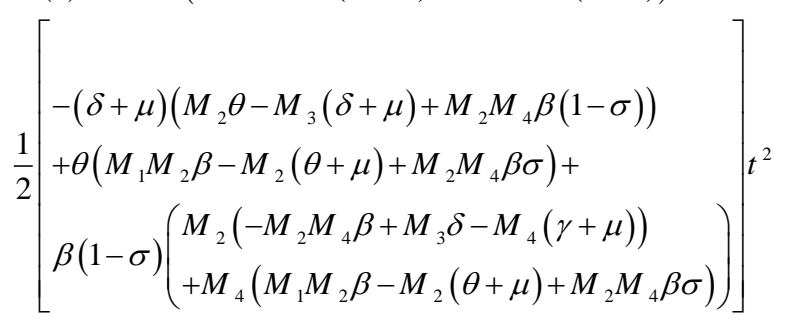

$C(t)=M_{4}+\left(-M_{2} M_{4} \beta+M_{3} \delta-M_{4}(\gamma+\mu)\right) t+$

$$
\frac{1}{2}\left[\begin{array}{l}
-(\gamma+\mu)\left(-M_{2} M_{4} \beta+M_{3} \delta-M_{4}(\gamma+\mu)\right) \\
+\delta\left(M_{2} \theta-M_{3}(\delta+\mu)+M_{2} M_{4} \beta(1-\sigma)\right) \\
-\beta\left(\begin{array}{l}
M_{2}\left(-M_{2} M_{4} \beta+M_{3} \delta-M_{4}(\gamma+\mu)\right) \\
+M_{4}\left(M_{1} M_{2} \beta-M_{2}(\theta+\mu)+M_{2} M_{4} \beta \sigma\right)
\end{array}\right)
\end{array}\right] t^{2}
$$

\section{NUMERICAL METHODS AND SIMULATIONS}

In this section, the numerical results are obtained based on the application of the (MDTM) to SIRC influenza model. Since most of the non-linear differential equations do not have exact analytic solutions, so approximation and numerical techniques must be used.

\subsection{Disease free equilibrium $\left(R_{0}=(\beta /(\mu+\theta))<1\right)$}

For numerical study, (for $\mathrm{R}_{0}<1$ ) the following parameters can be used as folow: 
$\mu=1 / 50 y^{-1}, \theta=73 y^{-1}, \delta=1 y^{-1}$

$\gamma=0.5 y^{-1}, \beta=50, \sigma=0.05$

This was done with the standard parameter values given above and initial values

$\mathrm{M}_{1}=0.8, \mathrm{M}_{2}=0.1, \mathrm{M}_{3}=0.04, \mathrm{M}_{4}=0.06$.

These values correspond to table 2 in [12].

By taking differential transform method to initial conditions is transformed as follows:

$\tilde{S}(0)=0.8, \tilde{I}(0)=0.1, \tilde{\mathrm{R}}(0)=0.04, \tilde{\mathrm{C}}(0)=0.06$.

.And from equations (18)-(21), The solution series can be easily written as follows :

$S(t)=\sum_{k=0}^{\infty} \tilde{S}(k) t^{k}=0.8-3.966 t+75.6219 t^{2}-929.833 t^{3}+$

$7823.46 t^{4}-34261 t^{5}-258727 t^{6}+8.4739 \times 10^{6} t^{7}-$

$1.22332 \times 10^{8} t^{8}+1.19552 \times 10^{9} t^{9}+\ldots$

$I(t)=\sum_{k=0}^{\infty} \tilde{I}(k) t^{k}=0.1-3.287 t+44.0704 t^{2}-137.968 t^{3}-$

$5355.53 t^{4}+112877 t^{5}-1.1166 \times 10^{6} t^{6}+3.15402 \times 10^{6} t^{7}+$

$9.4118 \times 10^{7} t^{8}-1.96714 \times 10^{9} t^{9}-\ldots$

$R(t)=\sum_{k=0}^{\infty} \tilde{R}(k) t^{k}=0.04+7.5442 t-129.199 t^{2}+$

$1188.38 t^{3}-3585.91 t^{4}-71322.8 t^{5}+1.3641 \times 10^{6} t^{6}-$

$1.22489 \times 10^{7} t^{7}+4.09274 \times 10^{7} t^{8}+6.14701 \times 10^{8} t^{9}-\ldots$

$C(t)=\sum_{k=0}^{\infty} \tilde{C}(k) t^{k}=0.06-0.2912 t+9.50631 t^{2}-$

$120.581 t^{3}+1117.98 t^{4}-7292.85 t^{5}+11224.9 t^{6}+$

$621014 t^{7}-1.27138 \times 10^{7} t^{8}+1.56927 \times 10^{8} t^{9}+\ldots$

In this section, Laplace transformation is applied to (24), which yields

$L\{S(t)\}=\frac{0.8}{s}-\frac{3.966}{s^{2}}+\frac{151.244}{s^{3}}-\frac{5579 .}{s^{4}}+\frac{187763 .}{s^{5}}-\frac{4.11132 \times 10^{6}}{s^{6}}$

$\frac{186283440}{s^{7}}+\frac{4.27085 \times 10^{10}}{s^{8}}-\frac{4.93243 \times 10^{12}}{s^{9}}+\frac{4.3383 \times 10^{14}}{s^{10}}+\ldots$

$L\{I(t)\}=\frac{0.1}{s}-\frac{3.287}{s^{2}}+\frac{88.1408}{s^{3}}-\frac{827.808}{s^{4}}-\frac{128533}{s^{5}}+\frac{1.35452 \times 10^{7}}{s^{6}}-$

$\frac{8.03952 \times 10^{8}}{s^{7}}+\frac{1.58963 \times 10^{10}}{s^{8}}+\frac{3.79484 \times 10^{12}}{s^{9}}-\frac{7.13836 \times 10^{14}}{s^{10}}+\ldots$

$L\{R(t)\}=\frac{0.04}{s}+\frac{7.5442}{s^{2}}-\frac{258.398}{s^{3}}+\frac{7130.28}{s^{4}}-\frac{86061.8}{s^{5}}-\frac{8.55874 \times 10^{6}}{s^{6}}+$

$\frac{9.82152 \times 10^{8}}{s^{7}}-\frac{6.17345 \times 10^{10}}{s^{8}}+\frac{1.65019 \times 10^{12}}{s^{9}}-\frac{2.23063 \times 10^{14}}{s^{10}}+.$.

$L\{C(t)\}=\frac{0.06}{s}-\frac{0.2912}{s^{2}}+\frac{19.0126}{s^{3}}-\frac{723.486}{s^{4}}+\frac{26831.5}{s^{5}}-\frac{875142 .}{s^{6}}+$

$\frac{8.08193 \times 10^{6}}{s^{7}}+\frac{3.12991 \times 10^{9}}{s^{8}}-\frac{5.1262 \times 10^{11}}{s^{9}}+\frac{5.69457 \times 10^{13}}{s^{10}}+\ldots$

For simplicity, replacing $s=(1 / t)$
$L\{S(t)\}=0.8 t-3.966 t^{2}+151.244 t^{3}-$ $5579 . t^{4}+187763 . t^{5}-4.11132 \times 10^{6} t^{6}-$

$186283440 t^{7}+4.27085 \times 10^{10} t^{8}-$

$4.93243 \times 10^{12} t^{9}+4.3383 \times 10^{14} t^{10}+\ldots$

$L\{I(t)\}=0.1 t-3.287 t^{2}+88.1408 t^{3}-$

$827.808 t^{4}-128533 t^{5}+$

$1.35452 \times 10^{7} t^{6}-8.03952 \times 10^{8} t^{7}+$

$1.58963 \times 10^{10} t^{8}+3.79484 \times 10^{12} t^{9}-4.3383 \times 10^{14} t^{10}+\ldots$

$L\{R(t)\}=0.04 t+7.5442 t^{2}-258.398 t^{3}+$

$7130.28 t^{4}-86061.8 t^{5}-8.55874 \times 10^{6} t^{6}+$

$9.82152 \times 10^{8} t^{7}-6.17345 \times 10^{10} t^{8}+$

$1.65019 \times 10^{12} t^{9}+2.23063 \times 10^{14} t^{10}+\ldots$

$L\{C(t)\}=0.06 t-0.2912 t^{2}+19.0126 t^{3}-$

$723.486 t^{4}+26831.5 t^{5}-875142 . t^{6}+$

$8.08193 \times 10^{6} t^{7}+3.12991 \times 10^{9} t^{8}-$

$5.1262 \times 10^{11} t^{9}+5.69457 \times 10^{13} t^{10}+\ldots$

padé approximant [4/4] of (26) and substituting $S$ can be obtained [4/4] in terms of S. Finally, by using the inverse Laplace transformation, the modified approximate solutions can be expressed as:

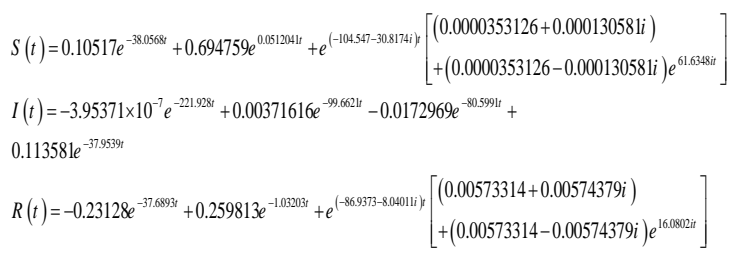

$C(t)=-7.1784 \times 10^{-6} e^{-132238 t}+0.0119018 e^{-39.554 t}+0.0483217 e^{3.83114 t}-0.000216349 e^{30.0015 t}$

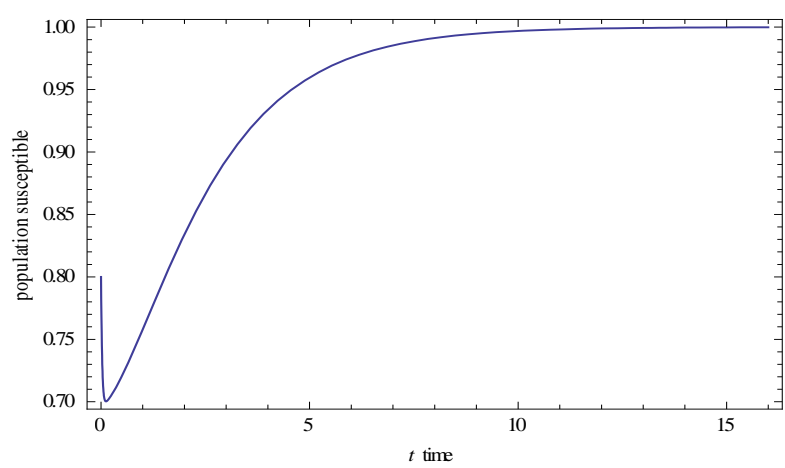

Figure1: $\mathrm{S}(\mathrm{t})$ for $\mu=0.02, \beta=50, \delta=1, \gamma=0.5, \sigma=0.05, \theta=73$ 


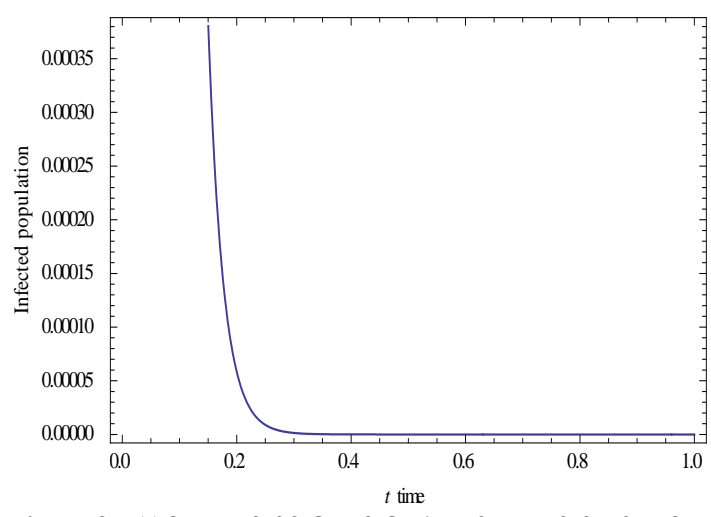

Figure2:I(t)for $\mu=0.02, \beta=50, \delta=1, \gamma=0.5, \sigma=0.05, \theta=73$

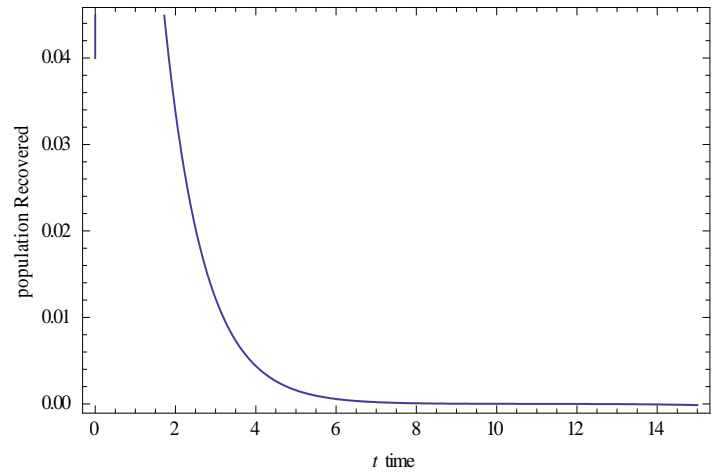

Figure3:R(t)for $\mu=0.02, \beta=50, \delta=1, \gamma=0.5, \sigma=0.05, \theta=73$

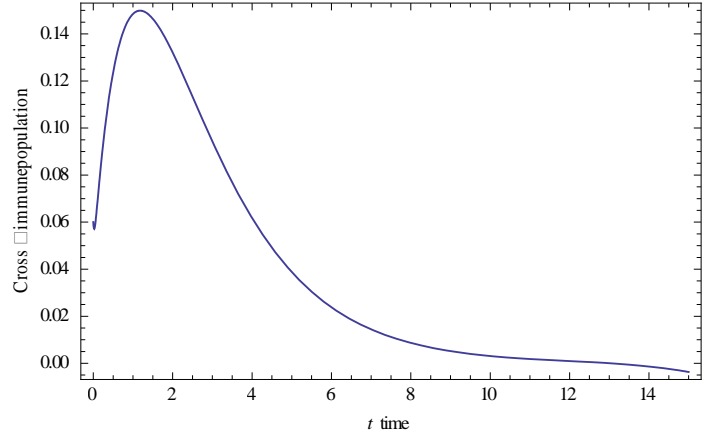

Figure4: $C(t)$ for $\mu=0.02, \beta=50, \delta=1, \gamma=0.5, \sigma=0.05, \theta=73$

\subsection{Endemic equilibrium}

$\left(R_{0}=\frac{\beta}{\mu+\theta}>1\right)$

In this section, to illustrate the capability of the MDTM, the variables and parameters, are considered as follows:

$\mu=1 / 50 y^{-1}, \theta=73 y^{-1}, \delta=1 y^{-1}$

$\gamma=0.5 y^{-1}, \beta=100, \sigma=0.05$

This was done with the standard parameter values given above and initial values

$\mathrm{M}_{1}=0.8, \mathrm{M}_{2}=0.1, \mathrm{M}_{3}=0.04, \mathrm{M}_{4}=0.06$.

These values correspond to table 2 in [12]. For the fourcomponent model. An approximation for $\mathrm{S}(\mathrm{t}), \mathrm{I}(\mathrm{t}), \mathrm{R}(\mathrm{t}), \mathrm{C}(\mathrm{t})$, the solution can be easily written as:

$$
\begin{aligned}
& S(t)=\sum_{k=0}^{\infty} \tilde{S}(k) t^{k}=0.8-7.966 t+10.6419 t^{2}+ \\
& 1153.98 t^{3}-5541.77 t^{4}-199026 t^{5}+ \\
& 1.80856 \times 10^{6} t^{6}+3.30018 \times 10^{7} t^{7}- \\
& 4.88965 \times 10^{8} t^{8}-4.91921 \times 10^{9} t^{9}+\ldots \\
& I(t)=\sum_{k=0}^{\infty} \tilde{I}(k) t^{k}=0.1+0.728 t-37.3279 t^{2}- \\
& 248.328 t^{3}+10102.4 t^{4}+51998.1 t^{5}-2.44897 \times 10^{6} t^{6}- \\
& 7.52897 \times 10^{6} t^{7}+5.59559 \times 10^{8} t^{8}+3.87404 \times 10^{8} t^{9}-\ldots \\
& R(t)=\sum_{k=0}^{\infty} \tilde{R}(k) t^{k}=0.04+7.8292 t+21.8457 t^{2}- \\
& 984.964 t^{3}-3838.52 t^{4}+158877 t^{5}+467477 . t^{6}- \\
& 2.71389 \times 10^{7} t^{7}-3.00545 \times 10^{7} t^{8}+4.71775 \times 10^{9} t^{9}-\ldots \\
& C(t)=\sum_{k=0}^{\infty} \tilde{C}(k) t^{k}=0.06-0.5912 t+4.84031 t^{2}+ \\
& 79.3108 t^{3}-722.135 t^{4}-11848.7 t^{5}+172935 t^{6}+ \\
& 1.66611 \times 10^{6} t^{7}-4.05398 \times 10^{7} t^{8}-1.85948 \times 10^{8} t^{9}+\ldots
\end{aligned}
$$

In this section, Laplace transformation is applied to the series solutions in (27), which yields

$$
\begin{aligned}
& L\{S(t)\}=\frac{0.8}{s}-\frac{7.966}{s^{2}}+\frac{21.2838}{s^{3}}+\frac{6923.88}{s^{4}}-\frac{133002}{s^{5}}- \\
& \frac{23883120}{s^{6}}+\frac{1.30216 \times 10^{9}}{s^{7}}+\frac{1.66329 \times 10^{11}}{s^{8}}- \\
& \frac{1.97151 \times 10^{13}}{s^{9}}-\frac{1.78508 \times 10^{15}}{s^{10}}+\ldots \\
& L\{I(t)\}=\frac{0.1}{s}+\frac{0.728}{s^{2}}-\frac{74.6558}{s^{3}}-\frac{1489.97}{s^{4}}+\frac{242458}{s^{5}} \\
& +\frac{6.23977 \times 10^{6}}{s^{6}}-\frac{1.76326 \times 10^{9}}{s^{7}}-\frac{3.7946 \times 10^{10}}{s^{8}}+ \\
& \frac{2.25614 \times 10^{13}}{s^{9}}+\frac{1.40581 \times 10^{14}}{s^{10}}+\ldots \\
& L\{R(t)\}=\frac{0.04}{s}+\frac{7.8292}{s^{2}}+\frac{43.6914}{s^{3}}-\frac{5909.78}{s^{4}}-\frac{92124.5}{s^{5}}+ \\
& \frac{1.90652 \times 10^{7}}{s^{6}}+\frac{336583440}{s^{7}}-\frac{1.3678 \times 10^{11}}{s^{8}}-\frac{1.2118 \times 10^{12}}{s^{9}}+ \\
& \frac{1.71198 \times 10^{15}}{s^{10}}+\ldots \\
& L\{C(t)\}=\frac{0.06}{s}-\frac{0.5912}{s^{2}}+\frac{9.68062}{s^{3}}+\frac{475.865}{s^{4}}-\frac{17331.2}{s^{5}}- \\
& \frac{1.42184 \times 10^{6}}{s^{6}}+\frac{1.24513 \times 10^{8}}{s^{7}}+\frac{8.39719 \times 10^{9}}{s^{8}}- \\
& \frac{1.63456 \times 10^{12}}{s^{9}}-\frac{6.74768 \times 10^{13}}{s^{10}}+\ldots
\end{aligned}
$$

For simplicity, replacing $\mathrm{s}=(1 / \mathrm{t})$ 
$L\{S(t)\}=0.8 t-7.966 t^{2}+21.2838 t^{3}+$

$6923.88 t^{4}-133002 t^{5}-23883120 t^{6}+$

$1.30216 \times 10^{9} t^{7}+1.66329 \times 10^{11} t^{8}-$

$1.97151 \times 10^{13} t^{9}-1.78508 \times 10^{15} t^{10}+\ldots$

$L\{I(t)\}=0.1 t+0.728 t^{2}-74.6558 t^{3}-$

$1489.97 t^{4}+242458 t^{5}+6.23977 \times 10^{6} t^{6}-$

$1.76326 \times 10^{9} t^{7}-3.7946 \times 10^{10} t^{8}+$

$2.25614 \times 10^{13} t^{9}+1.40581 \times 10^{14} t^{10}+\ldots$

$L\{R(t)\}=0.04 t+7.8292 t^{2}+43.6914 t^{3}-$

$5909.78 t^{4}-92124.5 t^{5}+1.90652 \times 10^{7} t^{6}+$

$336583440 t^{7}-1.3678 \times 10^{11} t^{8}-$

$1.2118 \times 10^{12} t^{9}+1.71198 \times 10^{15} t^{10}+\ldots$

$L\{C(t)\}=0.06 t-0.5912 t^{2}+9.68062 t^{3}+$

$475.865 t^{4}-17331.2 t^{5}-1.42184 \times 10^{6} t^{6}+$

$1.24513 \times 10^{8} t^{7}+8.39719 \times 10^{9} t^{8}-$

$1.63456 \times 10^{12} t^{9}-6.74768 \times 10^{13} t^{10}+\ldots$

padé approximant [4/4] is applied of (29) and substituting $t=\frac{1}{s}$, padé approximant [4/4] is obtained in terms of $\mathrm{S}$.

Finally, by using the inverse Laplace transformation, the modified approximate solutions can be expressed as:

$S(t)=1.04173 \times 10^{-6} e^{-1964.37 t}+$

$0.777741 e^{-8.17002 t}+e^{(-17.4076-59.9515 i) t}$

$\times\left[\begin{array}{l}(0.011129-0.010211 i)+ \\ (0.011129+0.010211 i) e^{119.903 i t}\end{array}\right]$

$I(t)=e^{(-2.08816-88.2489 i) t}$

$\times\left[\begin{array}{l}(0.00176682+0.000752284 i)+ \\ (0.00176682-0.000752284 i) e^{176.498 i t}\end{array}\right]+$

$e^{(0 .-22.6662 i) t}\left[\begin{array}{l}\left(\begin{array}{l}0.0482332+ \\ 0.00613219 i\end{array}\right) e^{3.36506 t}+ \\ \left(\begin{array}{l}0.0482332- \\ 0.00613219 i\end{array}\right) e^{(3.36506+45.3325 i) t}\end{array}\right]$

$R(t)=e^{(-1.19097-87.9418 i) t}$

$\times\left[\begin{array}{l}(-0.000457939+0.00163777 i)- \\ (-0.000457939+0.00163777 i) e^{175.884 i t}\end{array}\right]+$

$e^{0 .-22.5497}$

$\times\left[\begin{array}{l}(0.0204579+0.163655 i) e^{3.89398 t}+ \\ (0.0204579-0.163655 i) e^{(3.89398+45.0995 i) t}\end{array}\right]$

$C(t)=0.0608182 e^{-8.10172 t}+0.000185688 e^{70.5152 t}+$

$e^{(-2.51885-64.6573 i) t}$

$\times\left[\begin{array}{l}(-0.000501921-0.000882289 i)- \\ (-0.000501921-0.000882289 i) e^{129.315 i t}\end{array}\right]$

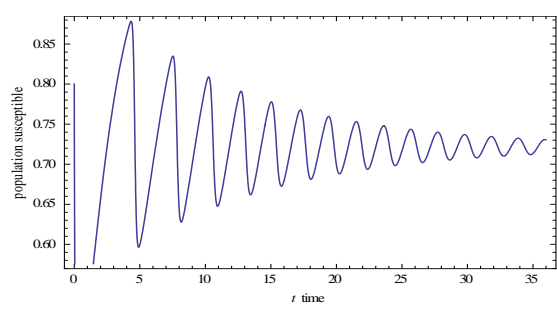

Figure5:S(t)for $\mu=0.02, \beta=100, \delta=1, \gamma=0.5, \sigma=0.05, \theta=73]$

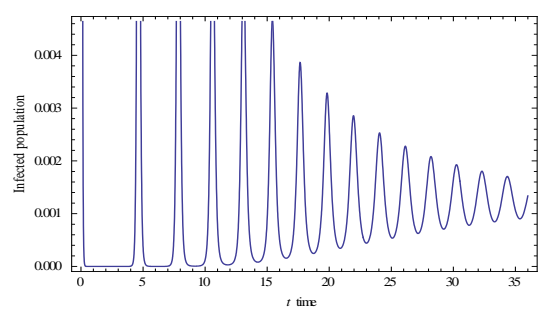

Figure6:I(t)for $\mu=0.02, \beta=100, \delta=1, \gamma=0.5, \sigma=0.05, \theta=73$ ]

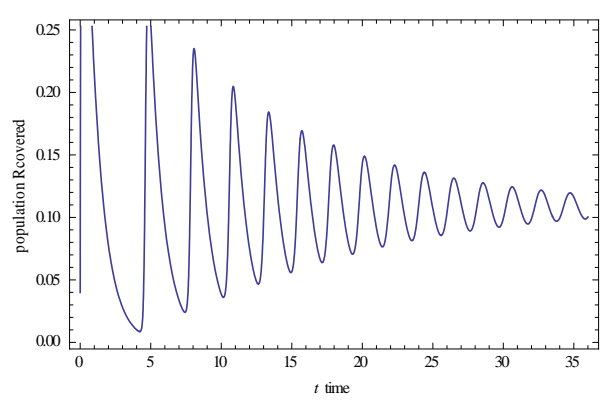

Figure $7: R(t)$ for $\mu=0.02, \beta=100, \delta=1, \gamma=0.5, \sigma=0.05, \theta=73]$

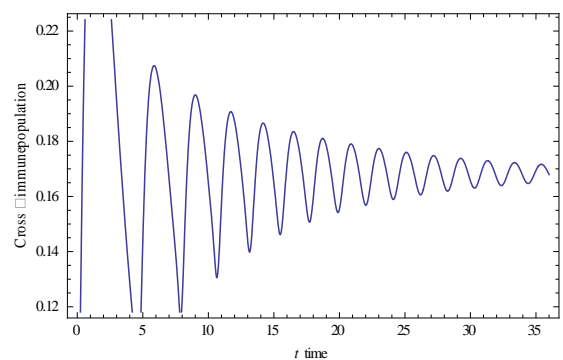

Figure8: $C(t)$ for $\mu=0.02, \beta=100, \delta=1, \gamma=0.5, \sigma=0.05, \theta=73]$

\section{CONCLUSIONS}

In this paper, the modified differential transform method has been used to obtain approximate analytical solution of nonlinear ordinary differential equation systems such as SIRC dynamical model. The accuracy and efficiency of this method was demonstrated by solving SIRC influenza model. Laplace transformation and padé approximant are used to obtain analytic solution and to improve the accuracy of differential transform method. The modified DTM is an efficient method for calculating periodic solutions of nonlinear differential equation system. The advantage of the method is that the approximate solutions can be calculated easily in shorter time with the computer programs such as Matlap and mathematica moreover this method solves the problem without any need for discretization, perturbation or linearization of the 
variables. The computations and graphs associated with the example in this paper were performed using Mathematica ver.8.

\section{ACKNOWLEDGMENTS}

The authors thank referees for fruitful comments and suggestions for revising the manuscript.

\section{REFERENCES}

[1] Palese, P. and Young, J. 1982. Variation of influenza A, $\mathrm{B}$, and C viruses. (Science 215)1468-1474.

[2] Casagrandi, R., Bolzoni, L., Levin, S.A., and Andreasen, V. 2006. The SIRC model and influenza A, Math. Biosci. (200) 152-169.

[3] Kermack, W. O. and McKendrick, A. G. 1927. Contributions to the mathematical theory of epidemics, Part I, Proc. Roy. Sot. Ser., A 115 , 700-721.

[4] Samanta, G. P. 2010. Global Dynamics of a Nonautonomous SIRC Model for Influenza A with Distributed Time Delay, Differ Equ Dyn Syst, 18 341-362.

[5] Jodar, L., Villanueva, R. J., Arenas, A. J., and Gonz'alez, G. C. 2008. Nonstandard numerical methods for a mathematical model for influenza disease, Mathematics and Computers in Simulation (79 ) 622-633.

[6] Zhou, J. K. 1986. Differential Transform and its Applications for Electrical Circuits, Wuhan, Huarjung University Press.

[7] Baker, G. A. 1975. Essentials of padé approximants. London: Academic press; .

[8] Chang, S. H., and Chang, I. L. 2008 A new algorithm for calculating one-dimensional differential transform of nonlinear functions. Appl Math Comput ;195:799-808.
[9] Jordan, D. W., and Smith, P. 1999. Nonlinear Ordinary Differential Equations. Oxford University Press.

[10] Dehghan, M., Shakourifar, M., and Hamidi, A. 2009. The solution of linear and nonlinear systems of Volterra functional equations using Adomian.Padé technique. Chaos Solitons Fract ;39:2509-21.

[11] Dehghan, M., Hamidi, A., and Shakourifar, M. 2007. The solution of coupled Burgers equations using Adomian.Padé technique. Appl Math Comput ; 189:1034-47.

[12] Chinviriyasit, W. 2007. Numerical modeling of the transmission dynamics of influenza, The First Inter. Symp. on Optim. and Sys. Biol., pp. 52-59.

[13] Pukhov, G.E. 1980. Differential transformations of functions and equations. Naukova Dumka, Kiev, (in Russian).

[14] Chen, S. S. and Chen, C. K. 2009 Application of the differential transformation method to the free vibrations of strongly non-linear oscillators, Nonlinear Analysis: Real World Applications 10 881-888.

[15] Yen-Liang Yeh, Cheng Chi Wang, Ming-Jyi Jang, 2007. Using finite difference and differential transformation method to analyze of large deflections of orthotropic rectangular plate problem. Appl. Math. Comput.; 190(2) 1146-1156.

[16] Abdel-Hialim Hassan, I. H. 2008 Application to differential transformation method for solving systems of differential equations Appl. Math Modelling ; 32(12)25522559 .

[17] Jang, M. J. and Chen, C. L. 1997. Analysis of the response of astrongly nonlinear damped system using a differential transformation technique. Appl. Math Comput.; 88(2-3): 137-151.

[18] Chen Chao-Kuang, Ho Shing-Huei. 1996. Application of differential transformation to eigenvalue problems. Appl Math Comput ; 79: 173-88. 